\title{
COMPARACIÓN DE LA SATISFACCIÓN LABORAL DEL DIRECTOR ESCOLAR Y LOS DOCENTES
}

\author{
Eileen Ramery-Gelpi \\ Universidad de Jaén. Andalucía, España \\ Dr. Eufrasio Pérez Navío \\ Universidad de Jaén. Andalucía, España
}

\begin{abstract}
Resumen. Este artículo es producto de un proyecto de investigación que tuvo como finalidad el comparar el grado de satisfacción laboral del director escolar con el del docente. Se evaluaron diferentes aspectos relacionados a la satisfacción laboral tales como: genero, edad, preparación académica, años de experiencia, deberes, autoridad, recursos, desarrollo profesional y ambiente institucional. El estudio se llevó a cabo en diferentes escuelas secundarias localizadas en el este de Orlando, Florida en los Estados Unidos de América. Una muestra de 293 docentes y 37 directores escolares fue seleccionada para participar en el estudio. La colección de datos fue mediante revisión bibliográfica, cuestionarios y entrevistas. Además, se utilizó la escala Likert para la cuantificación de los resultados. Los hallazgos de esta investigación reflejaron los niveles de satisfacción e insatisfacción existentes en directores escolares y docentes. Los aspectos de mayor importancia fueron la Autoridad, los Recursos, el Desarrollo Profesional y el Ambiente Institucional, ya que se encontró que la falta de comunicación, el pobre liderazgo para la toma de decisiones, el salario y la falta de recursos fueron la causa primordial del descontento e insatisfacción tanto en directores como en docentes. Como resultado del análisis realizado, una serie de recomendaciones fueron planteadas con el propósito de mejorar el desarrollo profesional y ambiente institucional, así como unas condiciones laborales que fomenten un proceso de enseñanzaaprendizaje de calidad. Cabe recalcar, que este estudio servirá para alentar a los miembros de la jerarquía educativa a encontrar medidas que fomenten un ambiente laboral que incite a docentes y directores a sentir una mayor satisfacción con la labor que realizan, para así lograr una educación de excelencia. La satisfacción del personal educativo fomentará un crecimiento en los diferentes aspectos de la educación, ya que la satisfacción laboral juega un papel importantísimo en el desempeño y la productividad de un individuo dentro de una organización.
\end{abstract}

Palabras clave: satisfacción laboral, insatisfacción laboral, docentes, directores, liderazgo.

\section{COMPARISON OF JOB SATISFACTION THE SCHOOL DIRECTOR AND TEACHER}

\begin{abstract}
This article is the product of a research project that aimed to compare the degree of job satisfaction of the school principal and the teacher.We evaluated different aspects related to job satisfaction such as: gender, age, academic qualifications, years of experience, duties, authority, resources, professional development, and institutional environment. The study was conducted in different secondary schools located in East Orlando, Florida. A sample of 293 teachers and 37 principals were selected to participate in the study. A sample of 293 teachers and 37 principals were selected to
\end{abstract}


participate in the study. Data collection was through literature review, questionnaires and interviews. Furthermore, the Likert scale to quantify the results was used. The findings of this research reflected the existing levels of satisfaction and dissatisfaction among school principals and teachers. The most important aspects were the authority, resources, professional development and the institutional environment, as it was found that poor communication, poor leadership decisions, wages and lack of resources were the primary cause of discontent and dissatisfaction for both principals and teachers. As a result of these considerations, a number of recommendations were raised in order to improve professional development and institutional environment and working conditions that foster a quality teaching-learning process. It should be noted that this study will encourage members of the educational hierarchy to find measures to promote a work environment that encourages teachers and principals to feel more satisfied with their work in order to achieve excellence in education. The satisfaction of the faculty will encourage growth in the different aspects of education, and job satisfaction plays an important role in the performance and productivity of an individual within an organization.

Keywords: job satisfaction, job satisfaction, teachers, principals, leadership.

\section{COMPARAÇÃO DE SATISFAÇÃO NO TRABALHO DIRETOR DE ESCOLA E PROFESSORES}

Resumo. Este artigo é o resultado de um projeto de pesquisa que teve como objetivo comparar o grau de satisfação do trabalho do professor e do diretor de escola. Nós avaliamos diferentes aspectos relacionados a satisfação do trabalho tais como: sexo, idade, habilitações literárias, anos de experiência, deveres, autoridade, recursos, desenvolvimento profissional e ambiente institucional. O estudo foi conduzido em diferentes escolas secundárias, localizadas ao leste de Orlando, Florida, nos Estados Unidos. Uma amostra de 293 professores e 37 diretores foi selecionado para participar no estudo. A coleta de dados foi através de revisão de literatura, questionários e entrevistas. Além disso, a escala de Likert para quantificar os resultados foi usada. Os resultados desta pesquisa refletiu os actuais níveis de satisfação e insatisfação com diretores de escolas e professores. Os aspectos mais importantes eram a autoridade, recursos, desenvolvimento profissional e do ambiente institucional, como verificou-se que a má comunicação, decisões de liderança pobres, salários e falta de recursos foram a principal causa descontentamento e insatisfação ambos os diretores e professores.Como resultado destas considerações, uma série de recomendações foram levantadas, a fim de melhorar o desenvolvimento profissional e ambiente institucional, e condições de trabalho que fomentem a qualidade do processo de ensino-aprendizagem. Note-se que este estudo irá incentivar os membros da hierarquia educacional para encontrar medidas para promover um ambiente de trabalho que incentive os professores e diretores a se sentirem mais satisfeitos com seu trabalho, a fim de alcançar a excelência em educação. A satisfação do corpo docente irá incentivar o crescimento nos diferentes aspectos da educação, uma vez que a satisfação no trabalho é fundamental no desempenho e produtividade de um indivíduo dentro de uma organização.

Palavras-chave: satisfação no trabalho, satisfação no trabalho, professores, diretores, liderança. 


\section{Introdução}

El ser humano, en su lucha por superarse más cada día, ha logrado enriquecer en gran manera su conocimiento pero al mismo tiempo se ha enfrentado a múltiples problemas que han influenciado grandemente su desempeño laboral. Al investigarse los niveles de satisfacción laboral entre directores escolares y docentes nos cuestionamos si existe alguna relación entre la satisfacción de ambos, en cuanto al puesto que ejercen y entre las similitudes y/o diferencias que existen en cuanto a los siguientes aspectos: deberes, autoridad, recursos, desarrollo profesional y ambiente institucional. En la actualidad, el auge que ha tomado el tema de la satisfacción laboral en las escuelas del este de Orlando, Florida en los Estados Unidos de América fue el factor motivante para investigar más a fondo este tema. La investigación fue centrada en las escuelas secundarias públicas de dicha zona. Este estudio profundiza en los factores causantes de la satisfacción y/o insatisfacción laboral del director escolar y el docente. Cabe recalcar, que todo esfuerzo con el propósito de mejorar los factores que influyen negativamente en la satisfacción pudiera saciar las carencias que existen actualmente dentro del plantel escolar.

\section{Concepto de satisfacción}

La variable satisfacción está vinculada con el concepto de motivación. Robbins (1999) define el concepto de satisfacción como "la voluntad de ejercer altos niveles de esfuerzo hacia las metas organizacionales, condicionadas por la habilidad del esfuerzo de satisfacer alguna necesidad individual." Continuando con el mismo autor, se puede decir que una necesidad es un estado interno de la persona que provoca que algunos resultados parezcan interesantes. Cuando una necesidad se encuentra insatisfecha crea tensión, lo cual estimula impulsos dentro del individuo. Estos impulsos hacen que el individuo busque metas particulares, que si se alcanzan satisfarán la necesidad y ayudarán a disminuir la tensión. Podemos concluir entonces que un trabajador realizará actividades motivado y con satisfacción si sus necesidades se conjugan con sus creencias y expectativas de realizar con éxito una tarea. Por otra parte, autores tales como González y González (2008) definen la satisfacción laboral como un sentimiento interno positivo del individuo, que está vinculado a la autoevaluación que el individuo realiza sobre su desempeño en un momento dado en el pasado y que se refleja en su conducta. De acuerdo a como estos autores definen la satisfacción, se puede llegar a la conclusión que el bienestar emocional juega un rol importantísimo en la conducta y desempeño de una persona en su ambiente de trabajo.

\section{Satisfacción del personal escolar y su importancia en el proceso de enseñanza aprendizaje}

En los años recientes, ha habido una proliferación de publicaciones de varios grupos de autores referente a la satisfacción profesional de una organización. La evidencia que atestigua a esto es la literatura existente sobre la satisfacción profesional y su consecuencia según (Aamodt 2004; Buitendach \& de Witte, 2005). Se ha 
encontrado que la insatisfacción laboral o profesional tiene una estrecha relación con los comportamientos tales como la despreocupación en el empleo, tardanza, ausentismo y productividad académica (Yousef, 2000). Sin embargo, la satisfacción laboral tiene una influencia en los empleados, la cual demuestra una propensión a permanecer con la organización y a superarse alcanzando niveles más altos (Buitendach \& de Witte, 2005).

Nos señala Griffin (2010) que el nivel de satisfacción laboral que un individuo experimenta puede tener un efecto significativo no solo en el individuo, sino también en quienes interactúan con él. La satisfacción profesional entre docentes es una construcción de múltiples facetas que es crítica y ha demostrado ser un determinante significativo para la retención del docente y alternadamente un contribuyente a la eficacia de la escuela. De la misma manera, tanto la actitud como la preparación del docente influyen significativamente en los resultados obtenidos en la conformación de la personalidad de los alumnos (Sarramona, 2008).

Güell (2014) menciona que los factores tales como salarios no competitivos, número de estudiantes asignados por docente, cantidad de horas lectivas y la autonomía de los centros, influyen considerablemente en la satisfacción laboral de los docentes. Por otra parte, Griffin (2010) señala además la importancia de estudiar aquellas variables que están ligadas a la satisfacción laboral para identificar las que pueden ser cambiadas o modificadas para dar lugar a una mayor satisfacción, motivación y bienestar por parte de los docentes en la labor que realizan. Según el autor, el conocer las distintas dimensiones que contribuyen a la satisfacción ayudará a tomar medidas que promuevan el mejoramiento de la misma. Aunque, podemos recalcar que existen dos actividades que brindan a los docentes una gran satisfacción como por ejemplo, su labor docente y su relación con el estudiantado. Por otra parte, Extremera et al. (2005) señala que muchos estudios han confirmado y evidenciado que el alto nivel de estrés que experimentan los docentes no solo afecta su propia salud física y emocional sino que influye de manera negativa el ambiente de enseñanza y los logros educativos.

\section{Teorías sobre la satisfacción}

Según Esteve (2009) la profesión docente es una actividad ambivalente. Esteve considera que algunos profesores disfrutan la enseñanza siendo esta el eje de su autorrealización personal, mientras, para otros la docencia es una fuente permanente de estrés capaz de romper su propio equilibrio personal. Durante sus treinta años de investigación su principal objetivo fue encontrar las claves de esta ambivalencia existente entre el personal docente. Además señaló que algunos de los aspectos negativos que afectan la profesión docente son: malestar, desgaste físico (burnout), bajas laborales de larga duración, desequilibrios psicológicos, etc. Esteve, 1997; Kyriacou, 1986, 1991 (citado en Esteve, 2009) nos permiten optimizar la actuación de nuestros profesores, dotándoles de recursos eficaces que rompen la ambivalencia y describen las fronteras que separan el éxito del fracaso en las aulas. Desde estos estudios, la formación inicial y continua se convierte en un análisis técnico de las 
conductas de los profesores, de los climas relacionales y emocionales que generan, de los códigos de comunicación que emplean en el aula y de sus estilos de respuesta ante la variedad de situaciones, que es necesario enfrentar en ese escenario multifactorial que es el aula.

\section{Estudios Relacionados}

Delgado (2012) nos presenta en su artículo "Las comunidades de liderazgo de centros educativos" las más novedosas y actuales vías de donde actualmente el tema del liderazgo institucional está siendo abordado. La propuesta de cultivar comunidades de líderes debe ser incluida en el trabajo colaborativo, la cual se expande y distribuye a todos los niveles con auténtica virulencia. La idea central de liderazgo comunitario trata de líderes, usualmente directivos de varios centros de adiestramiento que trabajan en colaboración para mejorar la calidad educativa, la cual debe ser reflejada en los resultados de aprendizaje de los alumnos de todos los colegios de una zona y en nuestra visión al futuro, como fondo de una auténtica reforma educativa en todos los niveles del sistema escolar.

Mulford (2006) señaló en su artículo "Liderazgo para mejorar la calidad de la educación secundaria: algunos desarrollos internacionales" que la escuela internacionalmente ésta en una edad de oro, la cual está ocurriendo en un ambiente de tremenda presión para que las escuelas rindan cuentas más públicamente. Esta mezcla de liderazgo y responsabilidad ha creado un contexto de trabajo muy nuevo para los docentes y líderes escolares. Hay una clara necesidad de entender mejor las consecuencias de este contexto para el trabajo de los líderes en las escuelas secundarias. Para ayudar a entenderlo, su artículo identifica algunas de las novedades internacionales en ese contexto de la educación y liderazgo escolar. Primero, se centra en trabajos que emanan de la OCDE (Organización para la Cooperación y el Desarrollo Económico) y luego, incluye novedades recientes en el Reino Unido y Australia. Este comienza en un nivel general, preguntando qué tipo de educación sirve mejor a la sociedad ahora y en el futuro. Luego, se examina la cuestión de cómo organizar mejor el sistema educativo para poder cumplir con las nuevas exigencias, sobre todo, en términos de su gobierno y liderazgo. Además, se identifican claramente tres áreas en este trabajo que tienen que ver con una ampliación de lo que se considera una buena enseñanza, un buen gobierno y un buen liderazgo escolar. Un segundo enfoque sobre revisiones recientes de la investigación sobre los efectos del liderazgo escolar en el aprendizaje del alumno, encuentra que coinciden con estos desarrollos internacionales y nacionales. También sugieren futuras directrices para un liderazgo eficaz en los centros escolares a nivel secundario.

Barroso (2005) nos indica en su artículo "Liderazgo y Autonomía de los Centros Educativos" las transformaciones que históricamente desde el siglo XX han acontecido en la regulación de las políticas educativas, modalidades de organización y gestión de las escuelas y como estas han afectado el trabajo de los directores escolares y como se 
ejerce el liderazgo escolar. Barroso, además destaca que ha habido una lenta evolución de una implantación de normas para regular los resultados, como el refuerzo de la autonomía de las escuelas y la generalización de los dispositivos de evaluación y/o rendimiento de cuentas. En la primera parte de su artículo, Barroso pone en evidencia las características del perfil tradicional de director escolar en el cuadro de una regulación burocrático-profesional, durante el siglo XX en Portugal, y en particular la oposición entre los roles de administrador y de líder profesional.

\section{Método}

El propósito de este estudio fue analizar la relación que existe entre el conocimiento que tiene el director escolar sobre los deberes y derechos de los docentes y la satisfacción de éstos con la labor que realizan. En este estudio se presentó la descripción de los siguientes tópicos relacionados con el problema: descripción de la población, descripción de la muestra, diseño de la investigación, descripción del instrumento, procedimiento de investigación y el análisis estadístico.

\section{Descripción de la población}

La población de éste estudio estuvo compuesta por 518 docentes y 37 directores escolares de la zona Este del condado de Orange en Orlando, Florida. El trabajo de investigación se llevó a cabo en las escuelas secundarias de dicha zona. La población de docentes estaba integrada por 390 docentes femeninos y 128 docentes masculinos y la población de directores estaba compuesta de 21 directores femeninos y 16 directores masculinos.

\section{Descripción de la muestra}

La muestra de este estudio consistió de 293 docentes, lo cual representó un cincuenta y dos por ciento (52\%) y 37 directores escolares que representaban el cien por ciento $100 \%$ de la población.

\section{Diseño de la investigación}

La investigación realizada fue una descriptiva-exploratoria. Datos fueron colectados mediante la exploración e indagación de la revisión bibliográfica, cuestionarios e entrevistas a personal docente y a directores escolares. A partir de los datos recolectados, se pudieron determinar y evaluar los factores que influyen de manera positiva y negativa a la satisfacción.

\section{Descripción del instrumento}

Para medir la relación existente entre el conocimiento que tiene el director escolar sobre los deberes y derechos de los docentes y la satisfacción de éstos en la labor docente se utilizaron dos cuestionarios: CADI (Cuestionario Administrado al Director) y el cuestionario CADO (Cuestionario Administrado al Docente), el cual es el suplemento del primer cuestionario (CADI). El Cuestionario Administrado al Director 
consistió de 47 ítems y se usó la escala Likert para la cuantificación de los resultados utilizando una escala del 5 al 1, donde 5 equivale a No Aplica, 4 equivale a Muy Satisfecho, 3 a Satisfecho, 2 a Insatisfecho y 1 a Muy Insatisfecho y para el segundo cuestionario CADO se utilizó la misma escala Likert pero fueron añadidos 4 ítems referentes a la satisfacción del docente.

Para este estudio se utilizó un cuestionario preparado por la investigadora, el cual fue revisado y evaluado por cuatro expertos en el desarrollo y construcción de instrumentos de investigación, los cuales aportaron mejoras al documento. Los contribuyentes del mejoramiento del documento fueron: el Doctor Andrés Santiago Mengual, docente de la Universidad de Alicante en España quien aportó unas mejoras muy valiosas; el Doctor Francisco Antonio Linares Lucerna, docente de Lengua Castellana y Literatura en el Instituto de Secundaria en España; el Doctor Juan Manuel Trujillo Torres, docente de la Universidad de Granada; y por último el Doctor José Luis Arboledas Nájera, docente de Música en el Instituto de Ciudad Real. El mismo se validó utilizando siete docentes y cuatro directores. Una vez sometidos los datos a un análisis estadístico de los cuestionarios mediante el programa "Statistical Package for Social Science" (SPSS), éstos resultaron con un coeficiente de confiabilidad de 0.936 para el cuestionario del docente y 0.854 para el cuestionario del director. Esto significa que los cuestionarios resultaron con una alta confiabilidad.

\section{Resultados}

En este estudio se presentaron los hallazgos según el orden de las preguntas de investigación y al final se presentó el resumen de los resultados obtenidos a través de los cuestionarios CADI y CADO.

Para identificar los ítems más importantes se utilizaron los promedios ponderados de cada ítem del total de los participantes. Para interpretar los resultados sobre los indicadores de satisfacción laboral se utilizó la siguiente escala de interpretación: 1.00 - 1.50 Muy Insatisfecho(a), 1.51 - 2.50 Bastante Insatisfecho(a), 2.51 - 3.50 Algo Insatisfecho(a), 3.51 - 4.50 Indeciso(a), 4.51 - 5.50 Algo Satisfecho(a), 5.51 - 6.50 Bastante Satisfecho(a), 6.51 - 7.00 Muy Satisfecho(a). Los resultados del análisis a las preguntas de investigación fueron los siguientes:

\section{Primera pregunta}

La primera pregunta fue la siguiente: ¿Cómo compara el Género del director escolar y el género del docente con su nivel de satisfacción laboral?

En los resultados obtenidos en el análisis comparativo según el género del director escolar y el docente, en el grupo de docentes del género femenino se observó que en los ítems de volumen de trabajo habitual, autonomía para planificar su trabajo, autonomía para implementar cambios, reconocimiento de su trabajo por parte de sus supervisores, forma en que se supervisa su trabajo, profesionalismo de parte de los 
colegas, oportunidad de utilizar sus destrezas y apoyo recibido por el superintendente de área obtuvieron los promedios más bajos. Por otra parte, el grupo de directores del género femenino se observó que los ítems con los niveles más bajos de satisfacción fueron: disponibilidad de recursos de instrucción, relaciones entre los directores y los docentes, reconocimiento recibido por colegas, respaldo recibido por su supervisor, reconocimiento recibido por el distrito y endoso de la administración hacia el docente.

En el análisis del grupo de docentes masculinos se observó que los ítems de volumen de trabajo habitual, autonomía para planificar su trabajo, autonomía para implementar cambios, reconocimiento de su trabajo por parte de sus supervisores, forma en que se supervisa su trabajo, profesionalismo de parte de los colegas, oportunidad de utilizar sus destrezas y apoyo recibido por el superintendente de área obtuvieron los promedios más bajos. Sin embargo, en el grupo de directores del género masculino se observó que los ítems de disponibilidad de recursos de instrucción, relaciones entre los directores y los docentes, reconocimiento recibido por colegas, respaldo recibido por su supervisor, reconocimiento recibido por el distrito y endoso de la administración hacia el docente obtuvieron los niveles más bajos de satisfacción.

En resumen, podemos llegar a la conclusión de que no existe diferencia significativa entre géneros, ya que tanto el género femenino como el masculino en ambos grupos mostraron sentirse insatisfechos en los mismos ítems.

\section{Segunda pregunta}

La segunda pregunta fue la siguiente: ¿Cómo compara la Edad del director escolar y el docente con su nivel de satisfacción laboral?

De acuerdo con los resultados obtenidos en el análisis comparativo de los 14 ítems según la edad del director escolar, existe un cierto grado de insatisfacción en los directores entre las edades de 36 a 40 años, en los ítems de reconocimiento de su trabajo por parte de sus superiores, disponibilidad de recursos de instrucción, relaciones entre los directores y los docentes, profesionalismo de parte de los colegas, reconocimiento recibido por colegas, respaldo recibido por su supervisor y reconocimiento recibido por el director escolar.

De igual manera, se observó que los directores de 41 a 45 años de edad tienen un porciento más alto de satisfacción en comparación con los docentes en los componentes de: volumen de trabajo, autonomía para planificar su trabajo, autonomía para implementar cambios, reconocimiento de su trabajo por parte de sus supervisores, forma en que se supervisa su trabajo, relaciones entre los directores y los docentes, profesionalismo de parte de los colegas y el apoyo recibido por el superintendente de área.

En el grupo de directores y docentes entre 46 a 50 años de edad se observó que los directores mostraron un nivel más alto de satisfacción laboral en los siguientes ítems: volumen de trabajo habitual, autonomía para implementar cambios, reconocimiento de 
su trabajo por parte de sus supervisores, forma en que se supervisa su trabajo, profesionalismo de parte de los colegas, reconocimiento recibido por el director escolar, oportunidad de utilizar sus destrezas y apoyo recibido por el superintendente de área.

Sin embargo, el grupo de docentes mostró un nivel de insatisfacción significativo en los mencionados ítems.

Por otra parte se observó además que los directores de más de 50 años de edad tienen el promedio más alto de satisfacción en los componentes de: volumen de trabajo, autonomía para planificar su trabajo, autonomía para implementar cambios, reconocimiento de su trabajo por parte de sus supervisores, profesionalismo de parte de los colegas y el reconocimiento recibido por colegas. Mientras, que el grupo de docentes mostro un nivel de satisfacción mayor en los ítems de: disponibilidad de recursos de instrucción, forma en que se supervisa su trabajo, relaciones entre los directores y los docentes y respaldo recibido por su supervisor.

\section{Tercera pregunta}

La tercera pregunta fue la siguiente: ¿Cómo compara la Preparación Académica del director escolar y el docente con su nivel de satisfacción laboral?

De acuerdo con los resultados obtenidos en el análisis comparativo de los 14 ítems según la Preparación Académica de los directores escolares y los docentes, existe un cierto grado de insatisfacción tanto en directores como docentes en el ítem de volumen de trabajo. Mas sin embargo, en los ítems de reconocimiento recibido por colegas, respaldo recibido por sus superiores, reconocimiento recibido por el distrito escolar, oportunidad de utilizar sus destrezas y endoso de la administración hacia el docente se observó una discrepancia bien marcada entre los directores y los docentes. Por otro lado, los ítems que obtuvieron los promedios más cercanos fueron volumen de trabajo, autonomía para implementar cambios, relaciones entre los directores y los docentes, profesionalismo por parte de los colegas y apoyo recibido por el superintendente de área.

En resumen, el director con una preparación académica más alta, siendo esta Doctorado, obtuvo un nivel mayor de satisfacción con un promedio de 4.52. Sin embargo, el director con un nivel académico de Maestría obtuvo un nivel de satisfacción más bajo que todos los demás grupos con un promedio de 4.13.

\section{Cuarta pregunta}

La cuarta pregunta fue la siguiente: ¿Cómo compara los Años de Experiencia del director escolar y el docente con su nivel de satisfacción laboral?

En los resultados obtenidos en el análisis comparativo de los 14 ítems según los años de experiencia del director escolar, se observó que los directores con 5 o menos años de experiencia obtuvieron los promedios más bajos. Estos ítems reflejan cierto grado de insatisfacción en los directores con las competencias de volumen de trabajo, autonomía para planificar su trabajo, autonomía para implementar cambios, 
disponibilidad de recursos, reconocimiento recibido por el distrito escolar, oportunidad de utilizar sus destrezas, endoso de la administración hacia el docente y el apoyo recibido por el superintendente de área. Mas sin embargo, este grupo obtuvo una mayor satisfacción con los ítems relacionados al reconocimiento por parte de sus supervisores $\mathrm{y}$ al profesionalismo por parte de sus colegas, con un promedio de 7.00.

En resumen, los directores con 5 o más años de experiencia obtuvieron el promedio de satisfacción más bajo de 4.00, a pesar de que obtuvieron los promedios más altos, en cuanto al reconocimiento por parte de sus supervisores y el profesionalismo por parte de sus colegas. El grupo con 6 a 10 años de experiencia obtuvieron 4.08 y el grupo de directores con 11 a 15 años de experiencia obtuvieron el nivel de satisfacción más alto con un promedio de 4.53 .

\section{Quinta pregunta}

La quinta pregunta fue la siguiente: ¿Cómo compara los aspectos de Deberes del director escolar y el docente con su nivel de satisfacción laboral?

En los resultados obtenidos en cuanto a los directores escolares en el análisis comparativo de como comparan los deberes del director escolar y el docente, se observó que todos los ítems tales como: competente en su profesión, resolución de conflictos, apoyo, profesionalismo, reconocimiento, iniciativa propia, falta de comunicación, autonomía en el desempeño de su labor, apoyo hacia un ambiente de motivación intrínseco, conceder autonomía y cooperación obtuvieron promedios por debajo de 3.50, reflejando cierto grado de insatisfacción.

\section{Sexta pregunta}

La sexta pregunta fue la siguiente: ¿Cómo compara los aspectos de Autoridad del director escolar y el docente con su nivel de satisfacción laboral?

De acuerdo con los resultados obtenidos en el análisis comparativo de los ítems de como compara la Autoridad del director escolar y el docente, se observó que en el grupo de docentes los ítems tales como: comunicación con los docentes de cada departamento, comunicación con los colegas y libertad de toma de decisiones en la institución, obtuvieron los promedios más bajos por debajo de 3.30, lo que refleja un cierto grado de insatisfacción. De igual manera, se observó que los directores obtuvieron un porcentaje más alto de satisfacción en comparación con el grupo de docentes.

\section{Séptima pregunta}

La séptima pregunta fue la siguiente: ¿Cómo compara los aspectos de Recursos del director escolar y el docente con su nivel de satisfacción laboral?

De acuerdo con los resultados obtenidos en el análisis comparativo de los ítems de como compara la satisfacción con el aspecto de recursos del director escolar y el docente, se observó que en el grupo de docentes el ítem de recursos financieros: 
discusión abierta del presupuesto y uso de los fondos obtuvo el promedio más bajo, por debajo de 1.50. Este ítem refleja un grado muy alto de insatisfacción por parte de todos los docentes.

De igual manera, se observó que los docentes mostraron tener un por ciento más alto de satisfacción en comparación con el director escolar en los ítems de recursos tecnológicos y multimedia y recursos de materiales necesarios para los docentes. Por otra parte, se observó que el promedio de satisfacción laboral más bajo fue la del grupo de docentes, el cual obtuvo un promedio de menos de 2.65 mostrándose algo insatisfecho.

\section{Octava pregunta}

La octava pregunta fue la siguiente: ¿Cómo compara los aspectos de Desarrollo Profesional del director escolar y el docente con su nivel de satisfacción laboral?

De acuerdo con los resultados obtenidos en el análisis comparativo de los ítems de como compara la satisfacción en el aspecto de Desarrollo Profesional del director escolar y el docente, se observó que en el grupo de docentes los ítems de reconocimiento como un competente y buen director escolar, reconocimiento de logros obtenidos, oportunidad de desarrollo profesional y oportunidad para involucrase con la comunidad, obtuvieron los promedios más bajos, por debajo de 2.80. Estos reactivos reflejan cierto grado de insatisfacción por parte de todos los docentes.

De igual manera, se observó que los directores mostraron tener un porcentaje más alto de satisfacción por encima de 2.80 en los siguientes: reconocimiento como un competente y buen director escolar, reconocimiento de logros obtenidos, oportunidad de desarrollo profesional, oportunidad para involucrarse a nivel de distrito y oportunidad para involucrarse con la comunidad.

Por otra parte, se observó que el promedio del grupo con el nivel de satisfacción laboral más bajo fue la del docente, el cual obtuvo un promedio de menos de 2.60 mostrándose algo insatisfecho.

\section{Novena pregunta}

La novena pregunta fue la siguiente: ¿Cómo compara los aspectos de Ambiente Institucional del director escolar y el docente con su nivel de satisfacción laboral.

De acuerdo con los resultados obtenidos en el análisis comparativo de los ítems de como compara la satisfacción con el aspecto de Ambiente Institucional del director escolar y el docente, se observó en el grupo de docentes que en los ítems de colaboración entre administradores y colaboración entre director y docente se obtuvo el promedio más bajo, por debajo de 2.80. Estos ítems reflejaron un cierto grado de insatisfacción por parte de todos los docentes.

De igual manera, se observó que los directores mostraron tener un porcentaje más alto de satisfacción por encima de 3.10 en los siguientes ítems: colaboración entre 
administradores, colaboración entre director y docente, colaboración entre directores, espíritu de cooperación entre compañeros, limpieza de la planta física, oportunidad de tener contacto con la comunidad, condiciones de trabajo y oportunidad de compartir experiencias educativas con los colegas.

Por otra parte, se observó que el promedio del grupo con el nivel de satisfacción laboral más bajo fue la del docente, el cual obtuvo un promedio de menos de 3.00 mostrándose algo insatisfecho.

\section{Discusión y conclusiones}

Esta investigación aportó evidencia empírica a las preguntas planteadas en el estudio, comprobando que sí existe una relación estrecha entre los factores de satisfacción laboral del director escolar y el docente. Los aspectos analizados en este estudio fueron el género, la edad, preparación académica, años de experiencia, deberes, autoridad, recursos, desarrollo profesional y ambiente institucional. Los hallazgos de este trabajo investigativo fueron más allá de lo esperado. El estudio demostró que docentes tanto en el género femenino como en el masculino están insatisfechos con el volumen de trabajo, la autonomía para la planificación de su labor, la autonomía para implementar cambios, el reconocimiento de su trabajo por parte de sus supervisores, la forma en que se supervisa su trabajo, el profesionalismo mostrado por parte de los colegas, la oportunidad de utilizar sus destrezas y el apoyo recibido por parte del superintendente de área. De igual manera, se demostró que directores de ambos géneros se mostraron insatisfechos con la disponibilidad de recursos de instrucción, las relaciones que existen entre los directores y los docentes, el reconocimiento recibido por sus colegas, el respaldo recibido por su supervisor(es), el reconocimiento recibido por el distrito y el endoso de la administración hacia el docente. Por lo tanto, podemos concluir que no hubo ninguna diferencia en el nivel de satisfacción según el género.

En cuanto a los años de experiencia de tanto el director como el docente se halló que existe una diferencia marcada entre los directores más jóvenes en edades de 36 a 40 años de edad, o sea con menos años de experiencia. Estos mostraron sentir una mayor insatisfacción en su labor. Mientras, directores con más de edad entre 41 años o más mostraron tener una mayor satisfacción, lo cual, nos demuestra que a más edad mayor satisfacción. Por otro lado, los docentes de 50 años o más fueron los que demostraron sentir una mayor satisfacción en la manera que se supervisa su labor, la disponibilidad de recursos, las relaciones entre los directores y colegas y el respaldo recibido por sus superiores.

Al analizarse los resultados sobre la preparación académica del director y el docente se halló que existen discrepancias en sus respuestas, ya que había una repetición en las respuestas de Algo Satisfecho e Indeciso. Pero si quedo demostrado que a mayor preparación académica mayor la satisfacción será.

Otro aspecto que se analizó en el estudio, fueron los años de experiencia tanto del director como del docente. Quedó demostrado que los años de experiencia juegan un 
papel importante en la satisfacción laboral. Los resultados evidenciaron que a mayor número de años de experiencia mayor será la satisfacción que tendrán tanto el director escolar como el docente.

En los aspectos de deberes se halló la insatisfacción que existe tanto en el director escolar como en el docente al demostrar que estaban insatisfechos en todos los ítems relacionados a los deberes. La excesiva demanda de deberes y responsabilidades, por parte de los administradores han sido algunos de los factores con más influencia. En las respuestas dadas por parte de los entrevistados se confirmó la frustración y el descontento que existe tanto en administradores escolares como en el personal docente y como está afectando negativamente su desempeño.

Al analizar los aspectos de Autoridad, Recursos, Desarrollo Profesional y Ambiente Institucional del director escolar y el docente, se encontró que la falta de comunicación, el pobre liderazgo para la toma de decisiones, el salario y la falta de recursos fue causa de descontento e insatisfacción para ambos.

Durante el estudio, además se observó una relación estrecha entre la cantidad exorbitante de deberes y responsabilidades y el ejercer un liderazgo de calidad. Es importante señalar que el liderazgo eficaz no solo es la capacidad de influir positivamente en otras personas para el logro de ciertas metas, sino que este debe ser ejercido con inteligencia, carisma, poder de convencimiento, sensibilidad, integridad, imparcialidad, innovación, y con buen tacto. Aunque el director escolar tenga el compromiso y la convicción para ejercer un liderazgo de calidad podemos exponer que el estrés físico y mental causado por el exceso de demandas y responsabilidades por parte de la jerarquía burocrática escolar influyen en los factores mencionados anteriormente, como los necesarios para ejercer un liderazgo eficaz.

La falta de comunicación entre el personal docente y el director escolar resultó además ser un factor que influyó grandemente en la insatisfacción laboral. Cabe recalcar, que esta falta de comunicación afecta grandemente el rendimiento académico de los estudiantes. Se estima que los resultados de esta investigación podrán ayudar en alguna medida a las escuelas del distrito escolar del Condado de Orange en Orlando, Florida, sirviendo de apoyo para tratar de reducir la insatisfacción laboral existente. El presente estudio pretende ser también un estímulo para el desarrollo de una línea de investigaciones en esta área, que además de incorporar nuevas variables, sus resultados permitan reducir las limitaciones señaladas. Se evidencia la importancia que tanto los docentes como los directores de escuela pública realicen acciones que tiendan a favorecer un ambiente de escuela acogedor y estimulante y que permita además la realización de estrategias metodológicas y la aplicación de elementos positivos. Esto ayudaría a enfrentar una educación de calidad en el presente siglo. Se estima que este trabajo podría tener un impacto significativo en el quehacer pedagógico en las escuelas públicas. 
Se considera que los resultados de esta investigación pueden contribuir a la predicción del rendimiento académico. Asimismo, ser un aporte de información sobre los factores que pueden ayudar a explicar la variabilidad de este producto educativo y así ayudar a reducir el fracaso en las escuelas públicas. Como era de esperar, todos los factores de la satisfacción laboral docente y de directores escolares han demostrado tener la misma importancia respecto al sentir del ambiente institucional. Se evidencia la importancia que tanto los directores como los docentes realicen acciones tendientes a favorecer un ambiente estimulante basado en reconocimientos. Por lo cual, se recomienda realizar actividades donde se le de reconocimiento tanto al director como al docente. De esta forma se desarrolla una de las necesidades de la jerarquía de Maslow, la necesidad de reconocimiento. A la vez, ésta brinda auto-reconocimiento, confianza, respeto y éxito, los cuales son factores que logran obtener una satisfacción laboral según Herzberg (1966), cuando nos presenta los factores motivacionales que tienen gran influencia en la satisfacción laboral. Por consiguiente, se deben planificar actividades tanto en las escuelas como fuera de las escuelas para brindar reconocimiento a los directores escolares y a los docentes. Algunas de estas actividades podrían ser: desayunos, almuerzos, asambleas, reconocimientos, otorgación de regalos o algo tan sencillo como flores, tarjetas, etc.

Se evidencia la necesidad de tener disponibles los recursos de instrucción necesarios para cubrir las necesidades del docente. Esto se pudiera lograr con la ayuda de padres voluntarios, empresas que adopten la escuela, donaciones y/o propuestas. Este factor tiene gran influencia en la satisfacción y la seguridad laboral del docente. Existe cierto consenso entre los investigadores, estos indican que el desarrollo profesional es de especial relevancia para el éxito en la labor docente. Es por eso, que se le debe brindar la oportunidad de poder participar en seminarios, conferencias, clases y charlas para así estar al día en cuanto a lo más reciente en la educación. Además, tener la oportunidad de asistir a eventos que enriquezcan la profesión y puedan luego compartir sus experiencias con sus compañeros docentes. Es necesario que el director escolar, tenga mayor comunicación con sus docentes y esté atento a las sugerencias planteadas en las reuniones realizadas, así como buscar las estrategias necesarias para elevar el grado de satisfacción en los docentes. Desde luego, es necesario buscar estrategias donde el docente pueda promocionarse de acuerdo a otro factor que no sea la antigüedad y donde se preste mayor atención a la labor profesional y a los resultados por este desempeño.

\section{Referencias}

Aamodt, M. G. (2004). Applied industrial/organizational psychology. Belmont, California: Wadsworth.

Barroso, J. (2005). Liderazgo y autonomía de los centros educativos. Revista Española
de
Pedagogía,
63(232).
Recuperado
de 
http://revistadepedagogia.org/2007060260/vol.-1xiii-2005/n\%C2\%BA-232septiembre-diciembre-2005/liderazgo-y-autonomia-de-centros-educativos.html

Bishay, A. (1996). Teacher motivation and job satisfaction: A study employing the experience sampling method. Journal of Undergraduate Sciences, 3(3). Recuperado de http://www.hcs.harvard.edu/ jus/0303/bishay.pdf

Buitendach, J. \& De Witte, H. (2005). Job insecurity, extrinsic, and intrinsic job satisfaction and affective organizational commitment of maintenance workers in a parastatal. South African Journal of Business Management, 36(2), 27-37.

Credé, M., Chernyshenko, O. S., Bagraim, J., \& Sully, M. (2009). Contextual performance and the job satisfaction-dissatisfaction distinction: examining artifacts and utility. Human Performance, 22(3), 246-272.

Delgado, M. (2012). Las comunidades de liderazgo de centros educativos. Revista Educar, 48 (1) 9-21.

De Pablos, J., González, T., \& González, A. (2008). El bienestar emocional del profesorado en los centros TIC como factor de innovación educativa. Revista Latinoamericana de Tecnología Educativa, 7(2), 45-55. Recuperado de http://relatec.unex.es/article/view/459

Duke, D. (1988). Why principals consider quitting. Phi Delta Kappan, 70(4), 308-312.

Esteve, J.M. (2009). La docencia: competencias, valores y emociones. Recuperado de http://www.oei.es/evp/PonenciaEsteve.pdf

Evans, L. (1998). The effects of senior management teams on teacher morale and job satisfaction: A case study of Rockville county primary school. Educational Management Administration and Leadership, 26 (4). Recuperado de http://ema.sagepub.com/content/26/4/417

Extremera, N., Montalbán, F. M., \& Rey, L. (2005). Engagement y burnout en el ámbito docente: Análisis de sus relaciones con la satisfacción laboral y vital en una muestra de profesores. Revista de Psicología del Trabajo y de las Organizaciones, 21(1-2), 145-158. Recuperado de http://www.redalyc.org/articulo.oa?id=231317039009

Griffin, D. K. (2010). A survey of Bahamian and Jamaican teachers' level of motivation and job satisfaction. Journal of Invitational Theory and Practice, 16, 57-77. Recuperado de http://files.eric.ed.gov/fulltext/EJ942558.pdf

Güell, L. (2014). Estudio de la satisfacción laboral de los maestros. (Tesis doctoral). Universitat Internacional de Catalunya, Barcelona, España. Recuperado de http://www.tdx.cat/bitstream/handle/10803/293783/Tesi_Luisa_Güell_Malet.pdf ?sequence $=1$

Herzberg, F. (1966). Work and the Nature of Management. Cleveland: The World. 
Howard Frederick Bull, I. (2005). The relationship between job satisfaction and organizational commitment amongst high school teachers in disadvantaged areas in the Western Cape. (Doctoral thesis). University of the Western Cape. Cape Town, South Africa. Recuperado dehttp://etd.uwc.ac.za/xmlui/handle/11394/267

Howell, J. P., Dorfman, P. W., \& Kerr, S. (1986). Moderator variables in leadership research. The Academy of Management Review, 11(1). Recuperado de http://amr.aom.org/content/11/1/88.abstract

Mulford, B. (2006). Liderazgo para mejorar la calidad de la educación secundaria: Algunos desarrollos internacionales. Revista de currículo y formación del profesorado, 10(1). Recuperado de http://www.ugr.es/ recfpro/rev101ART2.pdf

Robbins, S. (1999). Comportamiento organizacional. México: Ediciones Prentice Hall.

Sarramona, J. (2008). Formación del profesorado y carrera docente. Madrid, España: Santillana.

Shan, M. H. (1998). Professional commitment and satisfaction among teachers in urban middle schools. The Journal of Educational Research, 92(2), 67-73. Recuperado de http://www.tandfonline.com/doi/pdf/10.1080/00220679809597578

Steyn, G.M., \&Van Wyk, J.N. (1999). Job satisfaction: Perceptions of principals and teachers in urban black schools in South Africa. South African Journal of Education, 19(1), 37-43.

Yousef, D. A. (2000). Organizational commitment: a mediator of the relationships of leadership behavior with job satisfaction and performance in a non-western country. Journal of Managerial Psychology, 15(1), 6-24.

Fecha de recepción: 12/11/2015

Fecha de revisión: 12/11/2015

Fecha de aceptación: 27/01/2016 